\title{
Successful Treatment with Autologous Mesenchymal Stem Cells Therapy for Muscle Wasting Post-Surgical Repair of Achilles Tendon: A Case Report
}

\author{
Hassan Mubark \\ Rheumatologist, Institution: Auckland Regenerative Clinic, Ormiston Specialist Centre, 125 Ormiston Road, Flat Bush, \\ Auckland 2019, New Zealand
}

\begin{abstract}
Received Date: June 02, 2021; Accepted Date: June 09, 2021; Published Date: June 11, 2021
"Corresponding author: Hassan Mubark, Rheumatologist, Institution: Auckland Regenerative Clinic, Ormiston Specialist Centre, 125 Ormiston Road, Flat Bush, Auckland 2019, New Zealand.

Tel: +64 92713305/+64 21843513; Fax: +64 92770769; Email: drhassnmubark@ gmail.com
\end{abstract}

\section{Abstract}

Tendon rupture occurs when sudden forces apply upon the Achilles tendon during vigorous physical activities that involve abrupt pivoting on a foot or fast acceleration.

The initial management of Achilles tendon rupture consists of a non-operative approach, but if this fails, operational treatment should take place. This article presents a forty-four-year-old female patient who had a left Achilles tendon rupture during a netball game. Initially was treated conservatively, followed by re-rupturing it spontaneously, which required reconstruction surgery with a tendon transfer. Subsequently, she developed calf muscle weakness and atrophy at the grafted musculoachilles junction. Her manifestations were the inability to perform a single heel raise, impaired recreational activities, and calf muscle wasting and weakness. The Foot \& Ankle Disability Index (FADI) score was 74. She elected for a trial of autologous adipose-derived expanded mesenchymal stem cell therapy (MSCs) combined with platelet-rich plasma (PRP). Six months following the treatment, she had a good outcome evidenced by improvement of daily activities, performing heel-raise, and slow running for the first time after several years post reconstruction surgery. Her FADI score rose to 91.3 , and six months post-treatment MRI revealed increased signal at the musculo-achilles junction representing a possible healing process. This case suggests successful therapy outcome with a single MSCs and PRP, indicating regenerative therapy could be tried post Achilles rupture surgery when there is calf muscle wasting and weakness.

Keywords: Achilles tendon; Mesenchymal stem cells (MSCs); Platelet-rich plasma (PRP); Rupture; Surgery; Wasting

\section{Introduction}

Achilles tendon rupture is easy to diagnose by clinical examination, but an ultrasound scan (USS) enables rapid bedside confirmation of the diagnosis; magnetic resonance imaging (MRI) provides more anatomic details with greater accuracy and look for other pathologies.

Initial management of Achilles tendon rupture comprises rest, non-steroidal anti-inflammatory drugs, and ankle immobilization. If those were unsuccessful, then consider surgical intervention. Surgical repair diminished the risk of recurrent tendon rupture comparing with conservative management [1]. Operational treatment stemmed in a significantly higher complication rate than conventional treatment [2]. The increased complication risk may be associated with open repair surgery. Around eighty percent of athletes return to sports following Achilles tendon rupture [3].

Treatment of chronic Achilles rupture with flexor hallucis tendon transfer is a good option with a favorable outcome [4]. Despite an excellent surgical approach, long-term complications of scarring and some limitation of achieving competitive sports compared to pre-rupture status. There is growing evidence that MSCs can improve the tendon's function and repair [5].

\section{Case Report}

A Forty-four-year-old female patient works as a development Planner, usually fit and healthy; she ruptured her left Achilles tendon in March 2012 when she was playing netball; she felt as though someone kicked her in the back of the leg. She had conservative treatment with a standard leg cast, followed by moon boot, a non-weight-bearing, then weight-bearing until May 2012, and physiotherapy helped her recovery. In August2012, she felt a slight pull in her Achilles tendon, with a non-painful limp, so she did not do much about it. Ultrasound scan in the community revealed the Achilles tendon appears functionally ruptured with disorganized scar tissue in the tendon gap.

She has subsequently referred to the orthopedic surgeon for an assessment, and an MRI scan was obtained and showed complete rupture of the distal tendo-achilles. Moderate thickening at the myotendinous junction and some disuse edema within soleus, as in (Figure1). 


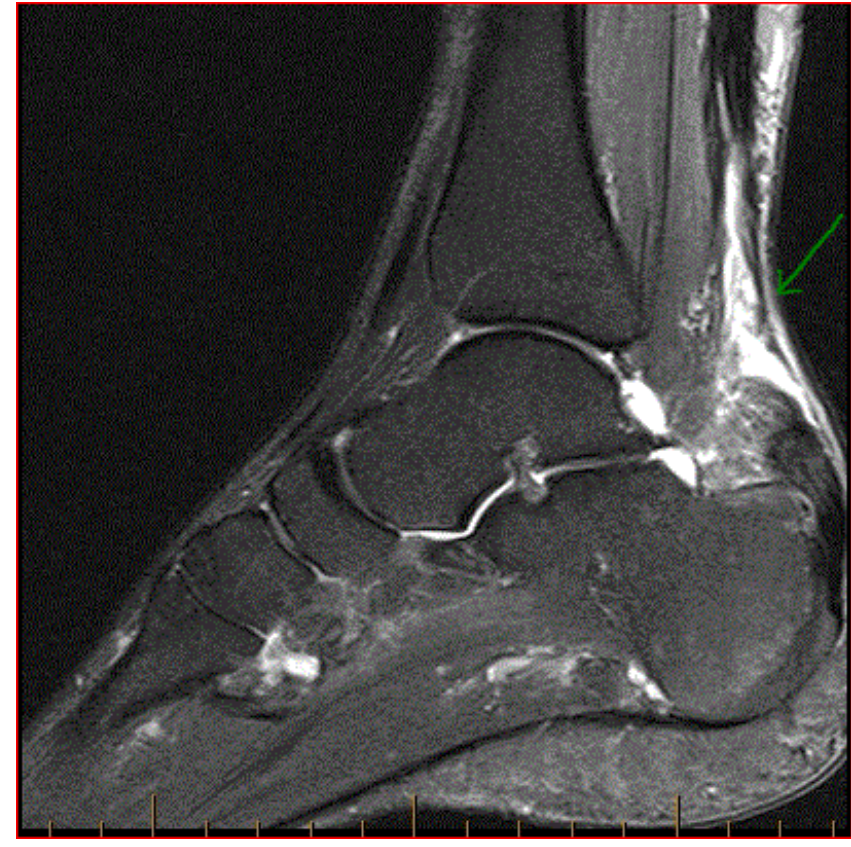

Figure 1: Sagittal T2 MRI Pre-surgery MRI demonstrating evidence of Achilles tendon rupture.

The surgeon felt it was difficult to stitch together the ruptured tendon. He recommended Achilles tendon reconstruction to reduce Achilles scar tissue and advised flexor hallucis longus tendon transfer in November 2012. She underwent several years of the rehabilitation program. Despite that, she developed calf muscle weakness and atrophy. In June 2020, the orthopedic surgeon noted calf muscle weakness and atrophy, and she could not perform a single heel raise. The Foot \& Ankle Disability Index (FADI) score of 74 and a follow-up MRI revealed remodeling of the left tendo-Achilles with surgical changes at the reconstruction site. There were tendinopathy/post-surgical changes in the distal tendoAchilles and soleus tendon insertion zones. There was a partial thickness tear at the soleus musculotendinous junction, as in (Figure 2).

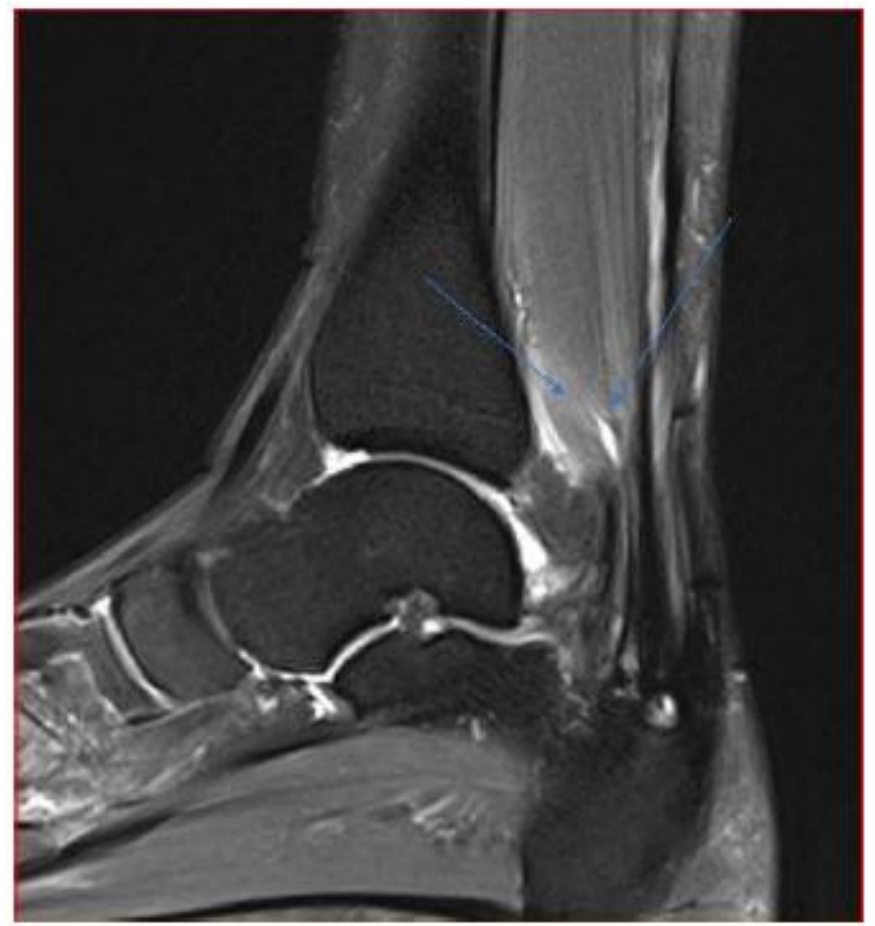

Figure 2: sagittal T2 MRI post-surgery muscle atrophy and partial thickness tear.

The surgeon advised her against any further surgical intervention, given it might provide only a modest effect at best. Because the gastric-soleus muscle complex was short for so long, the risk of surgery outweighs the benefit. She researched alternative therapy options, and she discovered a non-invasive treatment in New Zealand using adipose-derived expanded MSCs combined with PRP.
In August 2020, weassessed her at our regenerative clinic. The findings were post-surgery mid-calf scar with soleus muscle wasting at the tendo-muscular junction; she could not perform heel raise. She was concerned about her inability to carry out recreative sports or running. After discussing the pros and cons of the MSCs therapy, she elected to proceed with it.

On 21/10/2020, after informed consent, the abdominal 53 gram adipose tissue was harvested by a qualified general surgeon. According to the approved protocol, the MSCs were extracted and expanded at our licensed private Auckland lab, New Zealand. The lipo-aspirate was washed and then digested with $0.2 \mathrm{U} / \mathrm{mL}$ collagenases. The stromal vascular fraction (SVF) was separated from the digested adipose tissue through density centrifugation. The SVF was plated down and cultured using Dulbecco's Modified Eagle Medium (DMEM) and 10\% HPL to expand the MSCs population. Cells were grown to $90 \%$ confluence over eight weeks and then cryopreserved until the injection date. The implants got prepared on the date of treatment. The cells were washed and filtered before being resuspended in Hartmann's solution with 10\% HPL (human platelet lysate) in syringes for administration. Cell count was measured manually and confirmed by a hemocytometer, and the viability test was calculated by trypan blue exclusion dye, which ranged from $95-98 \%$.

On $16^{\text {th }}$ December 2020 , we performed the stem cell injections after informed consent. Under complete aseptic technique, we used $100 \times 10^{6}$ of MSCs $(2 \mathrm{~mL})$ combined with PRP (prepared on the day of the procedure, $8 \mathrm{ml}$ ); the PRP was prepared by using Anticoagulant Citrate Dextrose Solution -A (ACD-A) tubes and placed in a centrifuge for eight minutes. We added the PRP to the expanded stem cells. Total amount of $10 \mathrm{ml}$, we injected the solution in multiple areas of the soleus-achilles junction under ultrasound guidance, using a 1.5 -inch 25 Gauge. The injectate was distributed in a fanshaped fashion penetrating the scar tissues.

Six months post the stem cell implant, she reported a positive outcome clinically to heel raise and started to run slowly with the improvement of the FADI score to 91.3. An MRI in June2021 revealed an increased heterogeneous signal within the distal Achilles tendon. Stable atrophy and intramuscular edema within the calf together with slight increase in edema signal at the distal myotendinous junction of soleus, as in (Figure 3).

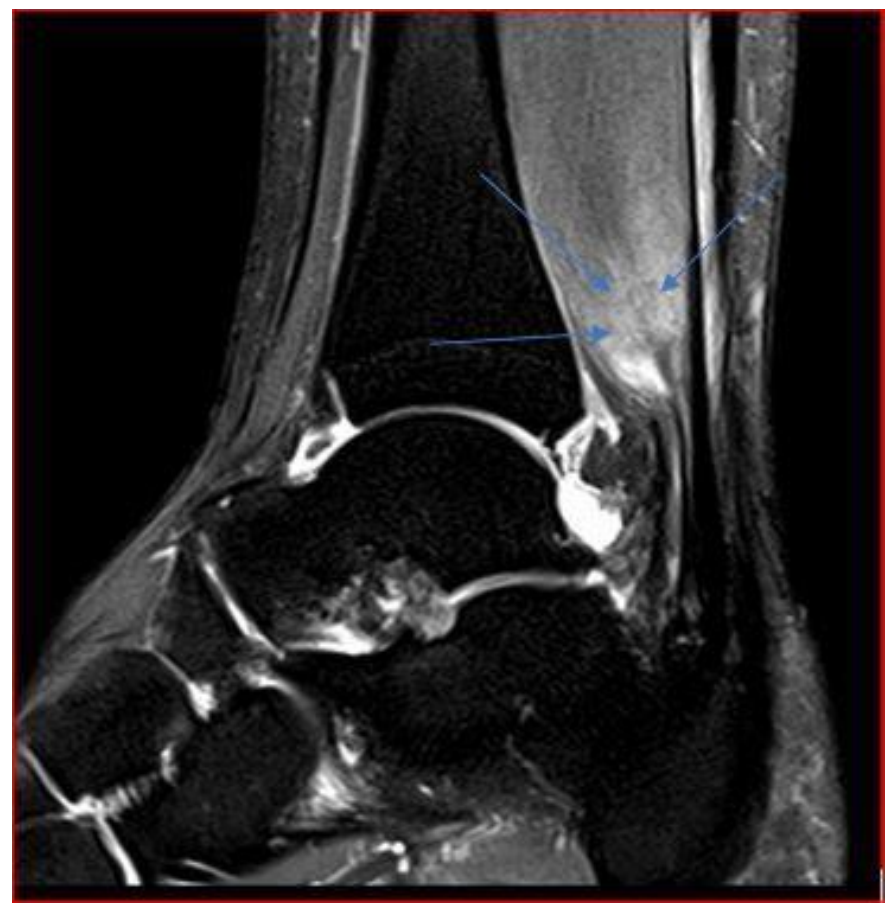

Figure 3: Sagittal T2 minor increase in signal may be due to post-treatment changes (healing response).

Discussion 
Some studies showed aggressive surgical intervention was the preferred option over conservative management. Based on early findings that conventional treatment is associated with high re-rupturerates. Regardless of the treatment modality, aggressive early rehabilitation is advocated for acute Achilles tendon ruptures to allow for an early return to activities of daily living, high patient satisfaction, and functional improvement.

There is growing interest in providing biological stimuli to heighten the tendon reparative response. MSCs are promising science as they have the potential to provide an appropriate cellular signal to promote new tendon formation (neotendon) during repair rather than scar tissue. This is currently being studied in various research facilities and clinical practices to ascertain efficacy and safety [6. 7].We believe the superb outcome in our patient could be related to a high number of MSCs combined with PRP in the same syringe for synergistic effect and implanted appropriately under ultrasound guidance in multiple areas with various depths at the affected musculoachilles junctions. The FADI score rose from 74 to 91.3 ; the improvements mainly came up to her toes and doing complex works. The MRI finding of increased signals six months posttreatment could be a good sign of healing, but it is worth repeating it in the future to see the progress.

We propose future randomized controlled trials to test this therapy and standardize the technique used for consistent results.

\section{Conflict of Interest}

The author confirms that this case study has not been previously published and has not been submitted or considered by any other journal. Additionally, all the contributors and clinicians have approved the contents of this case study.
The named author has drafted this case report and has no conflict of interest, financial or otherwise.

\section{References}

1. Wilkins R, Bisson LJ (2012) Operative versus nonoperative management of acute Achilles tendon ruptures: a quantitative systematic review of randomized controlled trials. Am J Sports Med 40: 154-160.

2. Jiang N, Wang B, Chen A, Dong F, Yu B (2012) Operative versus nonoperative treatment for acute Achilles tendon rupture: a meta-analysis based on current evidence. Int Orthop 36: 765-773.

3. Zellers JA, Carmont MR, GrävareSilbernagel K (2016) Return to play post-Achilles tendon rupture: a systematic review and meta-analysis of rate and measures of return to play. Br J Sports Med 50: 1325-1332.

4. Hahn F, Meyer P, Maiwald C, Zanetti M, Vienne P (2008) Treatment of chronic achilles tendinopathy and ruptures with flexor hallucis tendon transfer: clinical outcome and MRI findings. Foot Ankle Int 29: 794-802.

5. Almeida RC, Isabel C, Gomes ME (2019) Mesenchymal Stem Cells Empowering Tendon Regenerative Therapies. Int J Mol Sci 20(12): 2002.

6. Brown MH, Scholes C, Hafsi K, Marenah M, Li J, et al. (2019) Efficacy and safety of culture-expanded, mesenchymal stem/stromal cells for the treatment of knee osteoarthritis: a systematic review protocol. J OrthopSurg Res 14:34.

7. Centeno CJ, Al-Sayegh H, Freeman MD, Smith J, Murrell WD, et al. (2016) A multi-center analysis of adverse events among two thousand, three hundred and seventytwo adult patients undergoing adult autologous stem cell therapy for orthopaedic conditions. Int Orthop 40: 17551765.

Citation: Mubark H (2021) Successful Treatment WithAutologous Mesenchymal Stem Cells Therapy ForMuscle

Wasting Post-Surgical Repair of Achilles Tendon: A Case Report. Adv Ortho and Sprts Med: AOASM-145. 